\title{
Multi-Scale Investigation on Yield "Symmetry" and Reduced Strength Differential in an Mg-Y Alloy
}

\author{
Dalong Zhang, Lin Jiang, Xin Wang, M. Arul Kumar, Irene J. Beyerlein, \\ Julie M. Schoenung, Mo Li, Subhash Mahajan and Enrique J. Lavernia
}

\begin{abstract}
$\mathrm{Mg}$ and its alloys are promising candidates for light-weighted structural applications, e.g., aircraft, automobile, electronic, etc. However, the inherent hexagonal close packed crystal structure makes the deformation of $\mathrm{Mg}$ anisotropic, namely deformation only occur by dislocation slip in the close-packed (0001) plane (i.e., basal plane), or by deformation twinning in $\{10 \overline{1} 2\}$ planes. Consequently, polycrystalline $\mathrm{Mg}$ alloys undergone thermos-mechanical processing usually contain strong texture, i.e., preferred crystallographic orientation in grains. The texture in turn leads to anisotropic deformation in wrought $\mathrm{Mg}$ alloys. For example, in extruded $\mathrm{Mg}$ alloys, the compressive yield strength is usually much lower than the tensile yield strength (so-called yield asymmetry and strength differential). It is the anisotropy that hinders the broader application of $\mathrm{Mg}$ alloys. Recent modeling study on $\mathrm{Mg}$ predicts that certain alloying elements, particularly rare-earth elements (e.g., Y, Ce, $\mathrm{Nd}$, $\mathrm{Gd}$, etc.), could alter the active deformation modes, and promote more homogeneous deformation and overall mechanical properties in $\mathrm{Mg}$. Therefore, this work aims to investigate experimentally the effects of alloying element $\mathrm{Y}$
\end{abstract}

in reducing the intrinsic and extrinsic anisotropy, modifying texture, and enhancing the overall strength and ductility for $\mathrm{Mg}$. Fine-grained Mg 2.5 at.\% Y alloy (FG Mg-2.5Y) was prepared by powder metallurgy method, including gas atomization for producing $\mathrm{Mg}-2.5 \mathrm{Y}$ powder, degassing and hot isostatic pressing (HIP), and hot extrusion. Both the as-HIPed and the as-extruded materials were characterized by electron back-scattered diffraction (EBSD), transmission electron microscopy (TEM), and/or atom probe tomography (APT). Tension and compression tests were carried out along the extrusion direction (ED) for FG $\mathrm{Mg}-2.5 \mathrm{Y}$. Unlike common $\mathrm{Mg}$ alloys exhibiting yield asymmetry, the FG Mg$2.5 \mathrm{Y}$ exhibits virtual yield "symmetry" and significantly reduced strength differential. Namely the deformation is more isotropic. In addition to post-mortem TEM characterization for deformed FG $\mathrm{Mg}-2.5 \mathrm{Y}$, in situ TEM was also performed at the National Center for Electron Microscopy (NCEM), in an effort to understand the fundamental deformation mechanisms in FG Mg-Y that lead to reduced anisotropy. In situ TEM for single-crystal Mg-Y nano-pillars reveals that deformation twinning is replaced by dislocation slip in non-basal planes (i.e., prismatic planes), which diametrically differs from any other $\mathrm{Mg}$ alloys.
D. Zhang $(\bowtie) \cdot$ L. Jiang $\cdot$ X. Wang $\cdot$ J.M. Schoenung

E.J. Lavernia

Chemical Engineering and Materials Science,

University of California, Irvine, USA

e-mail: yzzhou@ucdavis.edu

M. Arul Kumar · I.J. Beyerlein

Theoretical Division,

Los Alamos National Laboratory, Los Alamos, USA
M. Li

Materials Science and Engineering,

Georgia Institute of Technology, Atlanta, USA

S. Mahajan

Chemical Engineering and Materials Science,

University of California, Davis, USA 